defined as good, and scores $\leq 80 \%$ as poor. Data from visits up to and including the visit before death were used; median follow-up was 38 months.

Because of missing data, 187 patients were excluded from the adherence analysis. Of those analyzed, 89\% showed good adherence and $11 \%$ showed poor adherence. Male sex was significantly associated with good adherence in both the candesartan and the placebo groups $(P=0.006$ and $P=0.004$, respectively), as was lower heart rate $(P=0.004$ and $P=0.001$, respectively). Good adherence was associated with lower all-cause mortality in both study groups $(P<0.0001$ for both).

The authors conclude that adherence to medication is independently associated with improved clinical outcome. As adherence to placebo also improved outcome, they propose that adherent behavior itself is associated with outcome, perhaps because medication-adherent patients are more likely to adhere to other effective treatments and behaviors.

Rebecca Ireland

Original article Granger BB et al. (2005) Adherence to candesartan and placebo and outcomes in chronic heart failure in the CHARM programme: double-blind, randomised, controlled clinical trial. Lancet 366: 2005-2011

\section{Myocardial infarction is associated with increased risk of subsequent stroke}

The risk factors and treatments for myocardial infarction (MI) and stroke are similar. Investigations have shown that the incidence of $\mathrm{Ml}$ is decreasing and survival after such an event is increasing; however, it is not known whether these changes also affect the incidence of stroke after index MI. Witt and colleagues examined the occurrence of ischemic and hemorrhagic stroke in a community-based cohort study of 2,160 patients who were hospitalized with incident $\mathrm{Ml}$ within the period 1979-1998.

The median follow-up was 5.6 years (range 0-22 years) and 273 strokes were recorded. The rate of stroke occurring within 30 days of the index $\mathrm{Ml}$ was 22.6 per 1,000 personmonths-a 44-fold increase relative to the expected rate for the general population, calculated from the Rochester Stroke Registry of the same community. In the first 3 years following $\mathrm{MI}$, the incidence of stroke was 2-3 times higher than in the general community, and the risk of stroke was increased with diabetes, increasing age and previous stroke. The risk of stroke after MI did not decline over the 19-year study period and the occurrence of stroke was associated with increased risk of death (adjusted hazard ratio 2.89; $95 \% \mathrm{Cl}$ 2.44-3.43).

The results show that treatments that have reduced the incidence of $\mathrm{Ml}$ do not decrease the risk of subsequent stroke. The use of antithrombotic interventions to prevent stroke should be considered, particularly in the 3 years immediately after Ml. The study cohort was mostly white and, therefore, these findings might not be generalizable to other ethnic populations.

Kate Matthews

Original article Witt BJ et al. (2005) A community-based study of stroke incidence after myocardial infarction. Ann Intern Med 143: 785-792

\section{Hyperglycemia is associated with poor outcomes in nondiabetics with acute MI}

Results from the French USIC 2000 study indicate that hyperglycemia on admission is a strong predictor of in-hospital and long-term mortality in nondiabetic patients with acute myocardial infarction (MI). Admission hyperglycemia has previously been shown to be predictive of outcome in diabetic patients with acute coronary syndromes.

Kadri et al. studied 1,604 nondiabetic patients in French intensive care units admitted for a recent MI (within 48 h) in November 2000. Raised admission glycemia (above the median of $6.9 \mathrm{mM}$ [124 mg/dl]) was associated with age older than 63 years, female sex, increased likelihood of ST-segment elevation $\mathrm{MI}$, and a raised frequency of reperfusion therapy. Hyperglycemic patients experienced more in-hospital complications and were more likely to develop cardiogenic shock following admission than those with glycemia $<6.9 \mathrm{mM}[<124 \mathrm{mg} / \mathrm{dl}]$. In-hospital mortality increased linearly and significantly in those with hyperglycemia, whereas 